\title{
Spatial-Structure of Fluctuation of Amount of Nanoparticles in Amplitude-Modulated VHF Discharge Reactive Plasma*)
}

\author{
Ren ZHOU, Kunihiro KAMATAKI, Hiroshi OHTOMO, Daisuke YAMASHITA, \\ Naho ITAGAKI, Kazunori KOGA and Masaharu SHIRATANI \\ Department of Electronics, Kyushu University, Fukuoka 819-0395, Japan
}

(Received 4 January 2019 / Accepted 16 March 2019)

\begin{abstract}
We have investigated spatial structure of fluctuation of amount of nanoparticles in amplitude modulated VHF discharge reactive plasma in order to understand interaction fluctuations between plasma and nanoparticles. The interaction fluctuation appeared in the whole discharge region. Especially, the strong interaction fluctuation existed at the edge region. The behaviors of time evolution of intensity of spatial structure of interaction fluctuation at the center and edge regions were different with each other. Nanoparticles at the edge region were smaller than ones in the main discharge region. It suggested that the interaction fluctuation is related to the growth of nanoparticles.
\end{abstract}

(c) 2019 The Japan Society of Plasma Science and Nuclear Fusion Research

Keywords: amplitude modulation, fluctuation, reactive plasma, nanoparticle, envelope analysis

DOI: $10.1585 / \mathrm{pfr} .14 .4406120$

\section{Introduction}

One of the important paradigm changes of semiconductor manufacturing is a transition from $2 \mathrm{D}$ to $3 \mathrm{D}$ architecture. With the increase of the number of stacked layers of 3D nanodevices, "3D-power scaling”, the transistors are arranged vertically rather than horizontally to increase the density without reducing the size of each transistor. Plasma processing is the main technology driver of this "3D-power scaling" in semiconductor device manufacturing [1]. Interaction fluctuations between reactive plasma and nanostructures have a great impact on such 3D nanodevice fabrication. As the number of stacked layers in 3D nanodevices increases, it is important to understand and control such interaction fluctuations [2-9]. Furthermore, plasma fluctuations themselves are classified as the core problem in plasma physics, because fluctuations are often found in various kinds of plasmas such as solar corona, magnetically confined plasma, quantum plasma, thermal plasma and processing plasma [10-15].

Among various plasma processing technologies, plasma enhanced chemical vapor deposition (PECVD) is one of important key technologies for semiconductor device manufacturing in the $3 \mathrm{D}$-power scaling era. In addition, CVD plasma usually contains nanoparticles which could change the properties of films [16-36]. In order to clarify the interaction fluctuations between reactive plasma and nanostructures, we have employed several methods such as an amplitude modulation (AM) method, an in-situ laser-light scattering (LLS) method and an envelope analysis [26-36].

author's e-mail: kamataki@plasma.ed.kyushu-u.ac.jp

*) This article is based on the presentation at the 2nd Asia-Pacific Conference on Plasma Physics (AAPPS-DPP2018).
Here, in order to investigate the relationship between the growth of nanoparticles and plasma fluctuations, we analyze spatial structures of the amount of nanoparticles and the interaction fluctuation between plasma and nanoparticles.

\section{Experimental}

We employed a capacitively coupled VHF discharge reactor with an LLS system, as shown in Fig. 1 [19-30]. $\mathrm{Si}\left(\mathrm{OCH}_{3}\right)_{2}\left(\mathrm{CH}_{3}\right)_{2}$ and $\mathrm{Ar}$ gas were supplied into the reactor at a flow rate of $0.2 \mathrm{sccm}$ and $40 \mathrm{sccm}$, respectively. The total pressure was 1.25 Torr. The temperature of the reactor wall was maintained at $373 \mathrm{~K}$ in order to avoid the liquefaction of $\mathrm{Si}\left(\mathrm{OCH}_{3}\right)_{2}\left(\mathrm{CH}_{3}\right)_{2}$. The electrodes were capacitively coupled via a matching unit to a $60 \mathrm{MHz}$ generator. The discharge power was $30 \mathrm{~W}$. The discharge

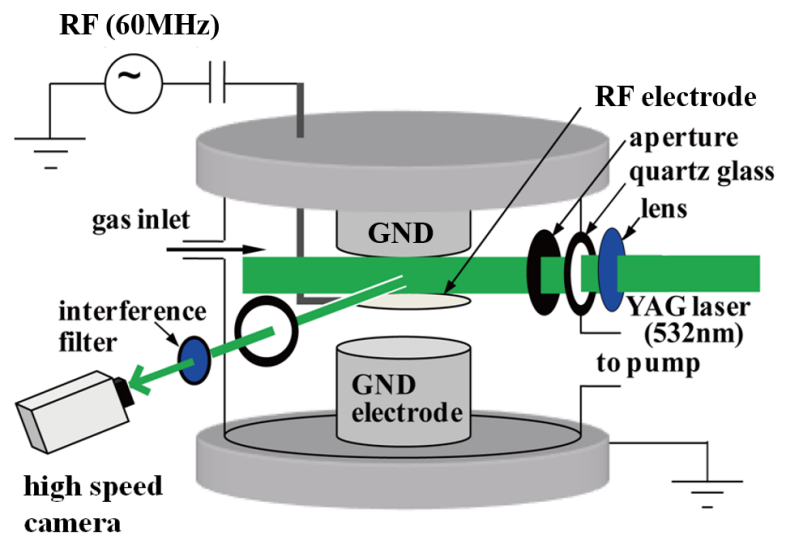

Fig. 1 Experimental apparatus. 
was sustained without modulation, or the discharge was sustained with an amplitude modulated VHF voltage with a sine waveform having a frequency of $100 \mathrm{~Hz}$ and the modulation level of $30 \%$ to perturb the plasma $\left(f_{\mathrm{AM}}=\right.$ $100 \mathrm{~Hz}, \mathrm{AM}$ level $=30 \%$ ). The discharge time $t_{\mathrm{on}}$ was $8 \mathrm{~s}$. The floating potential and Ar I $750 \mathrm{~nm}$ emission intensity, which is related to high energy electron density, fluctuated with a modulation frequency $[26,37]$. It means the fluctuation of plasma parameter can be controlled by AM of the VHF discharge voltage.

For obtaining information of nanoparticles size and density, we applied a two-dimensional laser light scattering (2DLLS) method [17, 19,28]. For this method, a sheet beam of $532 \mathrm{~nm}$ laser light was irradiated to the area between the electrodes (the diameter was $60 \mathrm{~mm}$ and distance between the electrodes was $20 \mathrm{~mm}$ ), and the light scattering intensity from the nanoparticles was measured by a high-speed camera equipped with an optical bandpass filter with a central wavelength $532 \mathrm{~nm}$. The bandwidth was $1 \mathrm{~nm}$ and the maximum transmission was $45 \%$. The LLS intensity was recorded at a frame rate of $1000 \mathrm{fps}$. The observed region was an area of $r=-35-35 \mathrm{~mm}$ and $z=3-17 \mathrm{~mm}$, where $(r, z)=(0 \mathrm{~mm}, 0 \mathrm{~mm})$ was the center of the upper surface of the powered RF electrode.

\section{Results and Discussion}

\subsection{Interaction fluctuation between plasma and nanoparticles}

\subsubsection{Spatial distribution of LLS intensity}

The scattered light is the Rayleigh scattering since the diameter of nanoparticles ranges from $2-15 \mathrm{~nm}$ which are much smaller than the laser light wavelength of $532 \mathrm{~nm}$. Therefore, the LLS intensity is proportional to the density $n_{\mathrm{p}}$ of nanoparticles and the sixth power of their size $d_{\mathrm{p}}$ [19]. Hereafter, the LLS intensity is referred to the amount of nanoparticles $\left(=n_{\mathrm{p}} \cdot d_{\mathrm{p}}^{6}\right)$. Therefore, the fluctuation of the LLS intensity is the fluctuation of the amount of nanopartilces. In the ref. [19], the growth rate of nanoparticles during discharge is $6.6 \mathrm{~nm} / \mathrm{s}$. The $6.6 \mathrm{~nm} \div 100=0.066 \mathrm{~nm}$, which is growth rate in the one period of AM frequency $(100 \mathrm{~Hz})$, is negligibly small. Therefore, the fluctuation of amount of nanoparticle imply the fluctuation of density of nanoparticles.

The fluctuation of amount of nanoparticles caused mainly by change of the density of nanoparticles. During one cycle of AM frequency, there are high and low discharge voltage periods since the discharge voltage fluctuates at the rate of sine wave. The high plasma density caused by high discharge voltage. This leads high generation ratio of nanoparticles. However, the low plasma density caused by low discharge voltage. This leads low generation ratio of nanoparticles. Therefore, fluctuation of discharge voltage leads the fluctuation of density of nanoparticles.
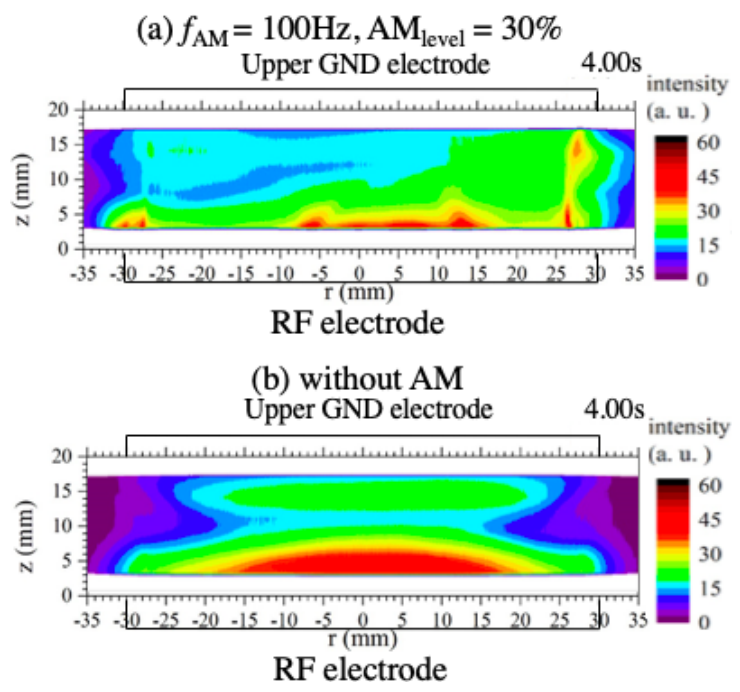

Fig. 2 Spatial profiles of LLS intensity of (a) $f_{\mathrm{AM}}=100 \mathrm{~Hz}$, $\mathrm{AM}_{\text {level }}=30 \%$ and (b) without $\mathrm{AM}$ at $4.00 \mathrm{~s}$.

Figures 2 shows the spatial distribution of LLS intensity (a) with $\mathrm{AM}$ of $f_{\mathrm{AM}}=100 \mathrm{~Hz}, \mathrm{AM}$ level $=30 \%$ and (b) without AM at $4.00 \mathrm{~s}$ after the discharge ignition at $z=3-17 \mathrm{~mm}$ and $|r|=0-35 \mathrm{~mm}$. The amount of nanoparticles is high near the plasma/sheath boundary region close to the VHF electrode with/without AM. However, the LLS intensity with AM is lower than that without AM at $|r|=0$ $20 \mathrm{~mm}$ which means that the growth of nanoparticles is suppressed by AM. Some nanoparticles reside around the edge region of the electrodes $(|r|=25-30 \mathrm{~mm})$ in the case of AM discharge, whereas nanoparticles without AM exist with a centrally symmetrical distribution.

\subsubsection{Interaction fluctuations of LLS intensity}

We extracted density fluctuation of nanoparticles form LLS intensity using the envelope analysis as follows. Firstly, we extracted a specific frequency component of LLS intensity, which is the response of the amount of nanoparticles to plasma disturbance. Next, we obtained its envelope which shows the interaction between plasma and nanoparticles at the specific frequency. Finally, we extracted the fluctuation of envelope which reflects the time evolution of the interaction fluctuation between plasma and nanoparticles [refer to Fig. 3]. A full description of the analysis method is reported in ref. [37]. We deduced spatial profiles of fluctuations of $100 \mathrm{~Hz}$ component of the amount of nanoparticles from LLS intensity as shown in Fig. 2.

Figures 3 shows the spatial structure of fluctuation of amounts of nanoparticles (a) with AM and (b) without AM. There is clear structure at the edge region in the case of with AM, while there is no such structure without AM. This result suggests that fluctuation of plasma parameter due to amplitude modulation affects fluctuation 


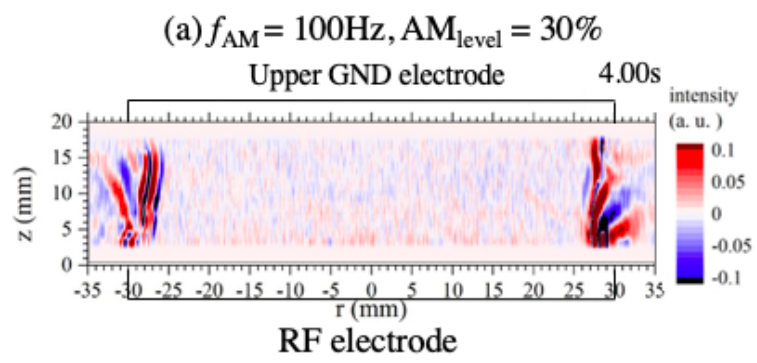

(b) without AM

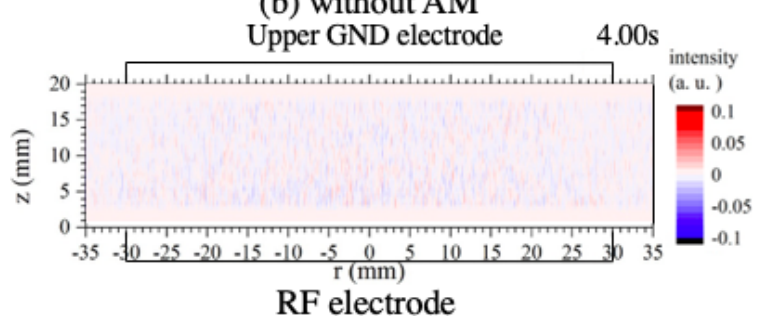

Fig. 3 Spatial profiles of interaction fluctuations of $100 \mathrm{~Hz}$ components of (a) $f_{\mathrm{AM}}=100 \mathrm{~Hz}, \mathrm{AM}_{\text {level }}=30 \%$ and (b) without $\mathrm{AM}$ at $4.00 \mathrm{~s}$.

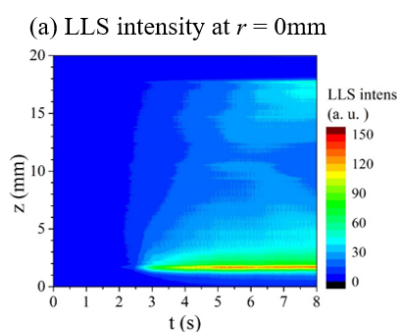

(c) LLS fluctuations of $100 \mathrm{~Hz}$ components at $r=0 \mathrm{~mm}$

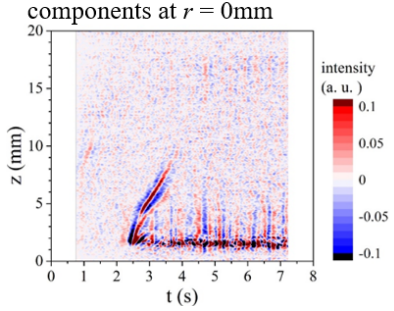

(b) LLS intensity at $r=27 \mathrm{~mm}$

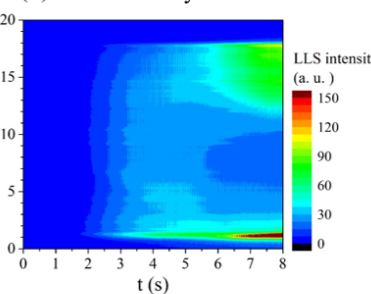

(d) LLS fluctuations of $100 \mathrm{~Hz}$ components at $r=27 \mathrm{~mm}$

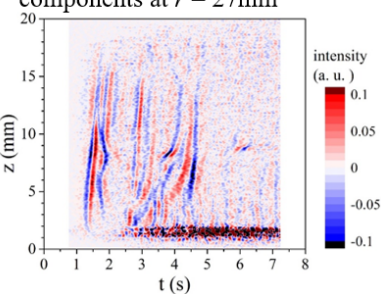

Fig. 4 Time evolution of spatial z-profiles of LLS intensity and the interaction fluctuation during the discharge around (a), (c) $r=0 \mathrm{~mm}$ and (b), (d) $r=-27 \mathrm{~mm}$.

of the amount of nanoparticles. It also implies interaction fluctuation between plasma and nanoparticles. There are the interaction fluctuation structures not only at the edge region but also in the whole discharge region in Fig. 4 (a). The intensity of interaction fluctuation in the whole discharge region is lower than at the edge region. This weak interaction fluctuation in the whole discharge region affects growth of nanoparticles, according to the results in Fig. 3 (a) and Fig. 4 (a). The suppression mechanism of growth of nanoparticle by AM method was illustrated in ref. [26] in details, which described as follows; the number of radicals for nucleation of nanoparticles increases with $\mathrm{AM}$, which decreases the radical flux to nanoparticles be- cause the ratio of increase of the number of nanoparticles is higher than that of radicals by AM. This leads to the suppression of nanoparticle growth and the generation of a high density of small nanoparticles.

\subsection{Interaction fluctuations and size distri- bution of nanoparticles}

\subsubsection{Spatial-structure of interaction fluctuations}

In order to analyze the relationship between the interaction fluctuation and the growth of nanoparticles, we deduce time evolution of spatial structure of the interaction fluctuation. Figures 4 shows the time evolution of spatial z-profiles of LLS intensity during the discharge around (a) $r=0 \mathrm{~mm}$ where is the center plasma region and (b) $r=-27 \mathrm{~mm}$ where is the edge plasma region. Nanoparticles are generated from $T_{\text {on }}>2 \mathrm{~s}$ in plasma sheath area both at the center and edge regions.

Figure 4 shows time evolution of spatial $z$-profile of the interaction fluctuation of $100 \mathrm{~Hz}$ components during discharge at (c) $r=0 \mathrm{~mm}$ and (d) $r=-27 \mathrm{~mm}$. The spatial $z$-profiles of the interaction fluctuation from $t_{\text {on }}>2 \mathrm{~s}$ at $r=0 \mathrm{~mm}$, and $t_{\mathrm{on}}>1 \mathrm{~s}$ at the edge region. Furthermore, the areas which the interaction fluctuations appear are different at these two positions. The interaction fluctuation at $r=0 \mathrm{~mm}$ appears at $t_{\mathrm{on}}=2-4 \mathrm{~s}$ between $z=2-8 \mathrm{~mm}$ and $t_{\mathrm{on}}>4 \mathrm{~s}$ between $z=2-4 \mathrm{~mm}$ where is plasma sheath region. The interaction fluctuation at $r=-27 \mathrm{~mm}$ appears at $t_{\text {on }}=2-5 \mathrm{~s}$ between $z=2-16 \mathrm{~mm}$ and $t_{\text {on }}>5 \mathrm{~s}$ between $z=2-10 \mathrm{~mm}$. The behavior of spatial structure of interaction fluctuation at the center and edge regions are different with each other. In the case of usual discharge without AM, there are no the spatial structure of interaction fluctuation of $100 \mathrm{~Hz}$ components (not shown).

\subsubsection{Size dispersion of nanoparticles}

To investigate the influence the difference of spatial structure of the interaction fluctuation between the center and edge regions on the growth of nanoparticles, the size dispersions were determined based on the LLS intensities $[19,28]$. Figure 5 shows the size dispersions at different radius (a) without AM and (b) with AM in each radical region. At first, these results suggest that the mean size of nanoparticle decreases by AM. Next, we focus on the size of nanoparticle in each radical region. In usual discharge (without AM), the average sizes at $r=0-10 \mathrm{~mm}$ and $r=10-20 \mathrm{~mm}$ are almost same $(\sim 18 \mathrm{~nm})$. However, in AM discharge, the average size $(\sim 11 \mathrm{~nm})$ at $r=0$ $10 \mathrm{~mm}$ and $r=10-20 \mathrm{~mm}$ where are the main discharge region is difference from ones $(\sim 8 \mathrm{~nm})$ at $r=20-30 \mathrm{~mm}$ where is the edge region. Nanoparticles at the edge region are smaller than those at the main discharge region in amplitude modulated discharge. The present results suggested that the interaction fluctuation is related to the growth of nanoparticles. Further studies are needed in order to clar- 

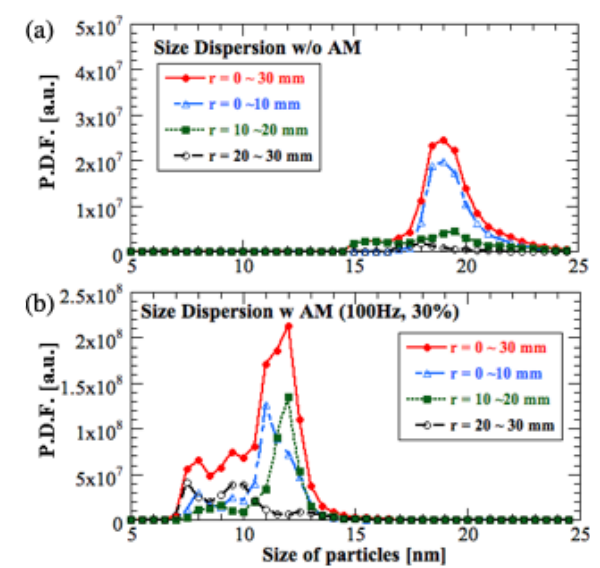

Fig. 5 Size dispersions of nanoparticles (a) without AM and (b) with AM in each radial region.

ify the relationship between size dispersion and interaction fluctuation of nanoparticle in edge regions.

\section{Summary}

We studied the spatial structure of LLS intensity and the interaction fluctuation between plasma and nanoparticles in amplitude modulated VHF discharge reactive plasma. Many nanoparticles exist at central plasma sheath region $(|r|=0-20 \mathrm{~mm})$ and around edge region of electrode $(|r|=25-30 \mathrm{~mm})$ in the case of AM discharge, whereas in the case of discharge without AM nanoparticles reside with a centrally symmetrical distribution. We deduced spatial profiles of interaction fluctuations of $100 \mathrm{~Hz}$ components of the amount of nanoparticles from LLS intensity using the envelope analysis. The interaction fluctuation appeared in the whole discharge region. Especially, strong interaction fluctuation existed at the edge region. The behaviors of time evolution of intensity of spatial structure of interaction fluctuation at the center and edge regions were different each other. To study the effect the difference of spatial structure of the interaction fluctuation between the center and edge regions on the growth of nanoparticles, the size dispersions were determined based on the LLS intensities. The nanoparticles at the edge region were smaller than ones at main discharge region. It suggested that the interaction fluctuation may be one of cause of the suppression mechanism. These results in this study will contributed to control the growth of nanoparticles and understand the interaction between plasma fluctuation and nanoparticles in nanodevice fabrications.

\section{Acknowledgments}

This work was partly supported by the JSPS KAKENHI Grant Number JP26246036 and the Collaborative Research Program of the Research Institute for Applied Mechanics, Kyushu University. The authors would like to thank Prof. Sanae-I Itoh, Prof. Shigeru Inagaki from Kyushu Univ. and Prof. Kimitaka Itoh, Dr. Tatsuya Kobayashi from NIFS for their helpful discussions and comments.

[1] ITRS 2.0, www.itrs2.net

[2] K. Ono, H. Ohta and K. Eriguchi, Thin Solid Films 518, 3461 (2010).

[3] W. Huang, M.R. Stan, S. Gurumurthi, R.J. Ribando and K. Skadron, 26th IEEE SEMI-THERM Symposium 198 (2010).

[4] K. Maex, M.R. Baklanov, D. Shamiryan, F. lacopi, S.H. Brongersma and Z.S. Yanovistskaya, J. Appl. Phys. 93, 8793 (2003).

[5] K.J. Kanarik, T. Lill, E.A. Hudson, S. Sriraman, S. Tan, J. Marks, V. Vahedi and R.A. Gottscho, J. Vac. Sci. Technol. 33, 020802 (2015).

[6] K. Ishikawa, K. Karahashi, T. Ichiki, J.P. Chang, S.M. George, W. Kessels, W.M.M. Kessels, H.J. Lee, S. Tinck, J.H. Um and K. Kinoshita, Jpn. J. Appl. Phys. 56, 06 HA02 (2017).

[7] T.W. Foresman, J. Appl. Phys. 46, 012055 (2016).

[8] M. Shiratani, K. Koga, S. Iwashita, G. Uchida, N. Itagaki and K. Kamataki, J. Phys. D 44, 174038 (2011).

[9] V.M. Donnelly and A. Kornblit, J. Vac. Sci. Technol. 31, 050825 (2013).

[10] G. Williams and I. Kourakis, Plasma Phys. Control. Fusion 55, 055005 (2013).

[11] M. Meyyappan, J. Phys. D 44, 174002 (2011).

[12] Y. Nagashima, K. Itoh, S-I. Itoh, A. Fujisawa, M. Yagi, K. Hoshino, K. Shinohara, A. Ejiri, Y. Takase, T. Ido, K. Uehara, Y. Miura and the JFT-2M group, Plasma Phys. Control. Fusion 49, 1611 (2007).

[13] M.A. Buldakov, V.A. Vershkov, M.Y. Isaev and D.A. Shelukhin, J. Phys.: Conf. Ser. 907, 012001 (2017).

[14] T. Ido, Y. Miura, K. Kamiya, Y. Hamada, K. Hoshino, A. Fujisawa, K. Itoh, S-I. Itoh, A. Nishizawa, H. Ogawa, Y. Kusama and JFT-2M group, Plasma Phys. Control. Fusion 48, S41 (2006).

[15] J. Cheng, L.W. Yan, K.J. Zhao, J.Q. Dong, W.Y. Hong, Q.W. Yang, X.T. Ding, X.R. Duan and Y. Liu, Nucl. Fusion 49, 085030 (2009).

[16] Y. Watanabe, M. Shiratani, Y. Kubo, I. Ogawa and S. Ogi, Appl. Phys. Lett. 53, 1263 (1988).

[17] M. Shiratani, H. Kawasaki, T. Fukuzawa, T. Yoshioka, Y. Ueda, S. Singh and Y. Watanabe, J. Appl. Phys. 79, 104 (1996).

[18] T. Fukuzawa, S. Kushima, Y. Matsuoka, M. Shiratani and Y. Watanabe, J. Appl. Phys. 86, 3543 (1999).

[19] S. Nunomura, M. Kita, K. Koga, M. Shiratani and Y. Watanabe, J. Appl. Phys. 99, 083302 (2006).

[20] K. Koga, S. Iwashita and M. Shiratani, J. Appl. Phys. D 40, 2267 (2007).

[21] S. Iwashita, K. Koga and M. Shiratani, Trans. Mat. Res. Soc. Jpn. 32, 501 (2007).

[22] S. Iwashita, M. Morita, K. Koga and M. Shiratani, J. Phys.: Conf. Ser. 100, 062006 (2008).

[23] S. Nunomura, M. Shiratani, K. Koga, M. Kondo and Y. Watanabe, Phys. Plasmas 15, 080703 (2008).

[24] M. Shiratani, K. Koga, S. Iwashita and S. Nunomura, Faraday Discuss. 137, 127 (2008).

[25] H. Miyahara, S. Iwashita, H. Miyata, H. Matsuzaki, K. Koga and M. Shiratani, Plasma Fusion Res. 8, 700 (2009).

[26] K. Kamataki, H. Miyata, K. Koga, G. Uchida, N. Itagaki 
and M. Shiratani, Appl. Phys. Express 4, 105001 (2011).

[27] K. Kamataki, K. Koga, G. Uchida, N. Itagaki, D. Yamashita, H. Matsuzaki and M. Shiratani, Thin Solid Films 523, 76 (2012).

[28] K. Kamataki, Y. Morita, M. Shiratani, K. Koga, G. Uchida and N. Itagaki, J. Instrum. 7, C04017 (2012).

[29] M. Shiratani, K. Koga, K. Kamataki, S. Iwashita, G. Uchida, H. Seo and N. Itagaki, Jpn. J. Appl. Phys. 53, 010201 (2014).

[30] M. Shiratani and K. Koga, J. Plasma Fusion Res. 90, 378 (2014).

[31] T. Ito, K. Koga, D. Yamashita, K. Kamataki, N. Itagaki, G. Uchida and M. Shiratani, J. Phys. Conf. Ser. 518, 012014 (2014).
[32] M. Shiratani, Y. Morita, K. Kamataki, H. Seo, G. Uchida, N. Itagaki and K. Koga, JPS Conf. Ser. 1, 015083 (2014).

[33] M. Soejima, K. Koga and M. Shiratani, Proc. IEEE-Nano 671 (2016)

[34] S. Toko, Y. Kanemitsu, K. Koga, H. Seo, N. Itagaki and M. Shiratani, J. Nanosci. Nanotechnol. 16, 10753 (2016).

[35] M. Shiratani, M. Soejima, H. Seo, N. Itagaki and K. Koga, Mater. Sci. Forum 879, 1172 (2017).

[36] S. Toko, Y. Torigoe, K. Keya, T. Kojima, H. Seo, N. Itagaki, K. Koga and M. Shiratani, Surf. Coat. Technol. 326B, 388 (2017).

[37] R. Zhou, K. Mori, H. Ohtomo, D. Yamashita, H. Seo, N. Itagaki, K. Koga and M. Shiratani, J. Phys.: Conf. Ser. (2018) in press. 